\title{
Increased IL17A, IFNG, and FOXP3 Transcripts in Moderate-Severe Psoriasis: A Major Influence Exerted by IL17A in Disease Severity
}

\author{
Priscilla Stela Santana de Oliveira, ${ }^{1}$ Michelly Cristiny Pereira, ${ }^{1}$ \\ Simão Kalebe Silva de Paula, ${ }^{1}$ Emerson Vasconcelos Andrade Lima, ${ }^{2}$ \\ Mariana Modesto de Andrade Lima, ${ }^{2}$ Rodrigo Gomes de Arruda, ${ }^{3}$ \\ Wagner Luís Mendes de Oliveira, ${ }^{1}$ Ângela Luzia Branco Pinto Duarte, ${ }^{2}$ \\ Ivan da Rocha Pitta, ${ }^{1}$ Moacyr Jesus Melo Barreto Rêgo, ${ }^{1}$ and Maira Galdino da Rocha Pitta ${ }^{1}$ \\ ${ }^{1}$ Laboratório de Imunomodulação e Novas Abordagens Terapêuticas (LINAT), Núcleo de Pesquisa em \\ Inovação Terapêutica Suely Galdino (NUPIT-SG), Universidade Federal de Pernambuco (UFPE), Recife, PE, Brazil \\ ${ }^{2}$ Hospital das Clínicas, Universidade Federal de Pernambuco (UFPE), Recife, PE, Brazil \\ ${ }^{3}$ Faculdade Nova Roma, Recife, PE, Brazil
}

Correspondence should be addressed to Maira Galdino da Rocha Pitta; mgrpitta@gmail.com

Received 19 July 2016; Revised 11 October 2016; Accepted 26 October 2016

Academic Editor: Yu Sun

Copyright (C) 2016 Priscilla Stela Santana de Oliveira et al. This is an open access article distributed under the Creative Commons Attribution License, which permits unrestricted use, distribution, and reproduction in any medium, provided the original work is properly cited.

\begin{abstract}
Psoriasis is a chronic and recurrent dermatitis, mediated by keratinocytes and T cells. Several proinflammatory cytokines contribute to formation and maintenance of psoriatic plaque. The Th1/Th17 pathways and some of IL-1 family members were involved in psoriasis pathogenesis and could contribute to disease activity. Therefore, we sought to analyse skin transcript levels of IL17A, IL22, RORC, IL8, IFNG, IL33, IL36A, FOXP3, and IL10 and correlate with clinic of patients with plaque-type psoriasis. In order to conduct that, we collected punch biopsies from lesional skin and obtained tissue RNA. After reverse transcription, qRT-PCR quantified the relative mRNA expression. The main results revealed increased transcripts levels of IL17A, IFNG, and FOXP3 in moderate-severe patients. Despite this, only IL17A can increase the chance to worsen disease severity. We also observed many significant positive correlations between each transcript. In conclusion, IL17A is elevated in lesional skin from psoriasis patients and plays crucial role in disease severity.
\end{abstract}

\section{Introduction}

Plaque-type psoriasis is the most prevalent form of psoriasis, corresponding to approximately $90 \%$ of cases. Erythematous scaly plaques, well demarcated, raised edges, and varied distribution throughout the body [1] characterize classical lesions. In some cases, systemic diseases such as inflammatory bowel disease and cardiovascular complications present worsening symptoms $[2,3]$.

The Th1/Th17 pathways are the principal immune components of the disease. The precise mechanism of how the plaque is formed remains uncertain, but when some kind of "triggers" are activated in skin, a cascade of molecules acts in the interaction between keratinocytes and immune cells. At the beginning of the process, interferon gamma (IFN $\gamma$ ) and tumor necrosis factor alpha (TNF $\alpha$ ) induce Langerhans cells to produce IL-12 and IL-23 [4]. IL-12 contributes to the strengthening of Th1 profile while IL-23 leads to Th17 differentiation that produces, mainly, IL-17 and IL-22 [5, 6]. In the epidermis, IL-8 operates as a potent chemotactic factor for neutrophils and contributes to the development of the erythema observed in skin lesions [7].

Recent studies have pointed out the active participation of others cytokines from IL-1 family. Between them there is 
IL-33, also named IL-1F11, that mediates biological functions through IL-1 orphan receptor ST2 [8]. In psoriasis, TNF $\alpha$ regulates IL-33, which promotes inflammation through mast and keratinocyte activation $[9,10]$. In addition, IL-36 $\alpha$ is one of the three homology proteins IL-36 $\alpha$ (IL-1F6), $\beta$ (IL1F8), and $\gamma$ (IL-1F9) that also belong to the IL-1 family. They are expressed in both dendritic and keratinocytes cells [11]. Evidence of the involvement of IL-36 cytokines in the pathophysiology of psoriasis includes the fact that a nonfunctional receptor antagonist (IL-36Ra) was associated with generalized pustular psoriasis [12]. Furthermore, IL-36 ligands deficient mice were protected from psoriasis form dermatitis model while the absence of IL-36Ra exacerbated the pathology [13].

On the other hand, regulatory $\mathrm{T}$ cells seem to fail in their peripheral anti-inflammatory control. Several researchers found decreased $\mathrm{CD}^{+} \mathrm{CD} 25^{+} \mathrm{FOXP}^{+} \mathrm{T}$ circulating cells both numerically and functionally in psoriasis patients $[14$, 15]. In order to balance the deficient anti-inflammatory response, clinical trials have been done using IL-10 recombinant human (rh) therapy protocols [16].

The aim of this study was to evaluate the pro- and anti-inflammatory psoriasis panel of molecules. Here, we sought to quantify Th17-related (IL17A, IL22, and RORC), Th1-related (IFNG and IL8), Treg-related (FOXP3 and IL10), and IL-1 family (IL33 and IL36A) skin transcripts and correlate with disease activity, systemic comorbidities, and methotrexate use in samples of Brazilian patients affected by psoriasis.

\section{Materials and Methods}

2.1. Ethics Committee. The human ethics committee of the Health Sciences Center of the Federal University of Pernambuco, located in Recife, Brazil, approved the study protocol (process number: 723.390).

2.2. Population Study. The study included twenty-one patients (11 male and 10 female) with plaque-type psoriasis attended and randomly selected in the Dermatology and Rheumatology Outpatient Clinic at Universidade Federal de Pernambuco. Only patients diagnosed with plaque-type psoriasis in strict accordance with the diagnostic criteria of Nestle et al. [17] with no prior immunobiologic therapy and no coexistent autoimmune disorders were considered. Psoriasis Area and Severity Index (PASI) was measured and classified as mild $(0-10)$ and moderate-severe $(>10)$ according to Menter et al. [18]. Other clinical parameters as comorbidities, disease duration, and previous systemic treatment were also questioned.

2.3. Skin Samples and RNA Extraction. Four milimeters ( $4 \mathrm{~mm}$ ) punch biopsies were taken from lesional skin of psoriatic patients. They were stored up to 24 hours at $4^{\circ} \mathrm{C}$ RNA later stabilization solution (Invitrogen Life Technologies, CA, EUA) until extraction. RNA was isolated using QIAGEN RNeasy Kit (Qiagen, Valencia, CA) and the amount was measured by nanodrop 2000 (Thermo Fisher Scientific, Carlsbad,
CA, EUA). The maximum of $500 \mathrm{ng}$ of total tissue RNA was reverse transcribed using High-capacity cDNA archive Kit 2X (Applied Biosystems Warrington, UK) according to manufacturer's instruction.

2.4. Quantitative Real-Time Polymerase Chain Reaction Analysis. qRt-pcr was carried out using predesigned Taqman probes gene expression assay (Applied Biosystems, Warrington, UK), using ABI Prism $7900 \mathrm{HT}$ sequence detection PCR machine (Applied Biosystems, Warrington, UK). We evaluated IL8 (Hs00174103_m1), IFNG (Hs00989291), IL-33 (Hs00369211_ml), IL36A (Hs00205367), IL17A (Hs00174383), IL22 (Hs01574154), IL10 (Hs00961622), RORC (Hs01076122 $\mathrm{ml}$ ), FOXP3 (Hs01085834_ml), and 18S (Hs03928990) as a housekeeping gene. The cycling condition consisted of 2 minutes at $50^{\circ} \mathrm{C}$ followed by 10 minutes at $95^{\circ} \mathrm{C}$. After these steps, there are 40 cycles at $95^{\circ} \mathrm{C}$ for 15 seconds and at $60^{\circ} \mathrm{C}$ for 1 minute.

2.5. Statistical Analysis. We used GraphPad PRISM 6.01 software (GraphPad Software Inc., San Diego, CA) and STATA 12 (StataCorp LP., Texas, USA) for data plotting and analysis. To ascertain the sample's normality, we performed D'Agostino \& Pearson omnibus normality test. The Mann-Whitney test and Spearman rank correlation were used when the variables did not follow Gaussian distribution. For variables that passed normality test, we applied unpaired $t$-test. We considered correlation $\left(R^{2}\right)$ strength as follows: $0<R^{2} \leq 0.35=$ weak correlation; $0.35<R^{2} \leq 0.67=$ moderate correlation; $0.67<$ $R^{2} \leq 1=$ strong correlation. To evaluate the association between transcripts levels and PASI, we performed multiple logistic regression for the clinical variables with dichotomous scores. The statistical significance was accepted when $p<$ 0.05 .

\section{Results}

3.1. Patients Cohort. A group of 21 patients, 11 men and 10 women, was included in this study. The mean age was 52 years with men showing a lower mean age than women (46.9 and 57.7, resp.). We stratified the PASI in accordance with the classification postulated by Menter and colleagues (2008), in the Journal of American Academy of Dermatology. PASI showed 8 as the lower punctuation and 28 as the highest score. General mean of PASI was $18 \pm 7.2$. According to that, men showed more severe disease (mean $21.3 \pm 5.8)$ than women $(14.4 \pm$ 7.2) and it was statistically significant, $p=0.026$. Thus, we wanted to know if the other clinical parameters interfered in disease activity. The findings showed that neither prior use of methotrexate nor presence or absence of comorbidities interfered in disease activity, $p=4823$ and $p=1182$, respectively (Data not shown). Table 1 details the clinical features of patients.

3.2. IL17A, IFNG, and FOXP3 mRNA Levels Were Increased in Skin Biopsy from Moderate-Severe Psoriatic Patients. We observed that in our sample only five individuals showed disease activity considered mild and the others had more 
TABLE 1: Clinical features of psoriatic patients from Brazil northeast ${ }^{\mathrm{a}}$.

\begin{tabular}{lc}
\hline Characteristics & All individuals $(n=21)$ \\
\hline Age (yrs.) & \\
$\quad$ Mean \pm SD/range & $52.05 \pm 13.48 / 23-74$ \\
Gender $N(\%)$ & $11(52.3)$ \\
$\quad$ Male & $10(47.7)$ \\
Female & $8.1 \pm 6.5(0.5-22)$ \\
Disease duration (years) & $9(42.8)-2.1 \pm 1.2$ \\
Mean \pm SD (range), all & $6(28.6)-9.1 \pm 1.3$ \\
$0-5$ years $N(\%)$-mean \pm SD & $6(28.6)-16.3 \pm 4.2$ \\
6-10 years $N(\%)$-mean \pm SD & \\
$>10$ years $N(\%)$-mean \pm SD & $5(23.8)-8.8 \pm 1$ \\
PASI clinical subgroups $N(\%)$ & $16(76.2)-20.8 \pm 5.7$ \\
Mild (PASI < 10)-mean \pm SD & \\
Moderate-severe (PASI $\geq 10)$-mean \pm & $3(14.3)$ \\
SD & $3(14.3)$ \\
Clinical comorbidities $N(\%)$ & $3(14.3)$ \\
Diabetes & $9(42.9)$ \\
Dyslipidemia & \\
Hypertension & \\
Treatment $N(\%)$ & \\
Methotrexate & \\
\hline
\end{tabular}

${ }^{a}$ Considering a Gaussian distribution, clinical values were represented by mean \pm SD

severe clinical presentation. As we can see in Figures 1(a), 1(b), and $1(\mathrm{c})$, only three of the nine transcripts analysed showed statistical significance in moderate-severe disease compared with mild activity. IL17A, IFNG, and FOXP3 showed $p=$ $0.0192, p=0.0237$, and $p=0.0239$, respectively. Figures $1(\mathrm{~d}), 1(\mathrm{e}), 1(\mathrm{f}), 1(\mathrm{~g}), 1(\mathrm{~h})$, and $1(\mathrm{i})$ provide the other analysed transcripts graphs.

3.3. Correlation between Cytokines in Skin Biopsies from Psoriatic Patients. After analysing the PASI score and their influence in expression of a panel of both pro- and antiinflammatory transcripts, we evaluated the importance that each cytokine could play over the expression of the others. We summarized the significances and correlation's coefficients in Table 2.

In our study, we identified several statistically significant positive correlations between the molecules analysed in different magnitudes. With $p<0.05$, IL17A correlated with its own transcription factor, RAR-related orphan receptor C (RORC), and other cytokines, such as IL22 and IL8. We also correlated IFNG versus IL10 and FOXP3; IL22 versus IL8 and IL10; RORC versus IL10; and lastly IL8 versus IL10.

We observed more significant correlation $(p<0.01)$ between IL36A versus IL17A, IFNG, IL8, and IL10. Similarly, FOXP3 showed correlation with IL10 and IL17A with IFNG. Strongly significant correlation $(p<0.001)$ was observed between the two transcription factors RORC versus FOXP3.
This last one also correlated with IL36A and IL17A. Finally, IL10 showed significant correlation with IL17A.

3.4. Men and Women Have Different Profile of Cytokines Expressed in the Skin. As previously mentioned, men and women exhibited different gravity of disease. Therefore, we investigated whether this fact influences the cytokine expression in the skin, separating the groups by gender. We observed that the male group showed significant high levels of IL17A transcripts compared to woman as well as more severe disease (Figures 2(a) and 2(b)). However, there was no significant statistical when we correlated each other (Figure 3(a)). In contrast, it occurred in female group. IL17 A and FOXP3 transcripts showed significant statistical positive correlation with PASI (Figures 3(b) and 3(c)).

Among the fifteen statistically significant correlations described above, eight correlations only remained in the male group. They were IL17A versus, IL8, IL22, IL36A, and IL10, IL10 versus IL8 and IL22, IL36A with IL8, and RORC versus FOXP3. In the opposite, three correlations occurred only in female group: IL17A versus IFNG and FOXP3 and this last one with IL36A (Table 3).

3.5. IL17A Exhibits Greater Influence on Disease Severity. Finally, in order to understand the relationship between transcripts levels and PASI score, we conducted a multiple logistic regression. We found that IL17A high levels increased the chance to have moderate-severe disease as shown in Table 4. Due to the multicollinearity existence, IFNG and IL10 could not be included in the regression model.

\section{Discussion}

Recently, we demonstrated that IL-17A, IL-22, and IL6 cytokines were more elevated in serum from psoriasis patients than in heathy controls. However, we have not found any correlation between systemic cytokine expression and disease severity [19]. So, we decided to investigate the lesion microenvironment and investigate if there was a correlation with disease severity and if it occurred in a local level in our group.

Over the past years, researches focused in Th1/Th17 pathways and described high levels of IFN $\gamma$, IL-17, and its isoforms in lesional psoriatic skin in both gene and protein levels [20-23]. Despite the availability of studies of psoriasis large-scale genomic and transcriptomes platforms [24-26], there is still a gap between the current knowledge and the clinical progression.

Our study showed increased expression of IL17A, IFNG, and FOXP3 in patients who exhibited severe clinical disease profile. Just as we did, Kim and Colleagues (2016) found significant difference in IL17A transcript expression between their clinically stratified groups [27]. In contrast to our study, they showed that patients classified with mild disease activity had the highest levels of IL17A. Suárez-Fariñas et al. (2012) also detected greater expression of IL23 p19 and p40 subunits IL17, IL22, and IFNG in lesions of moderate-severe patients using real-time reverse transcriptase method [28]. 


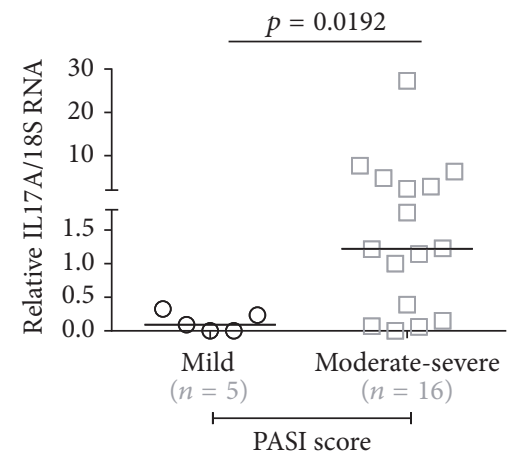

(a)

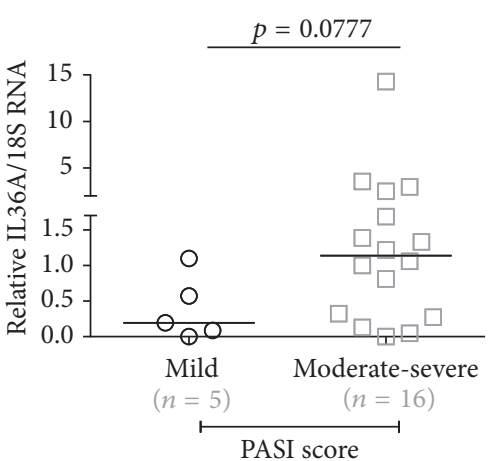

(d)

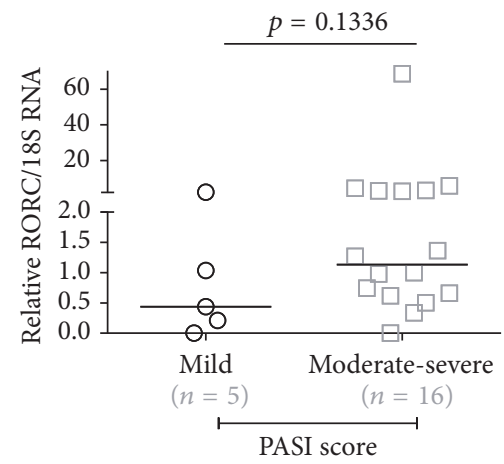

(g)

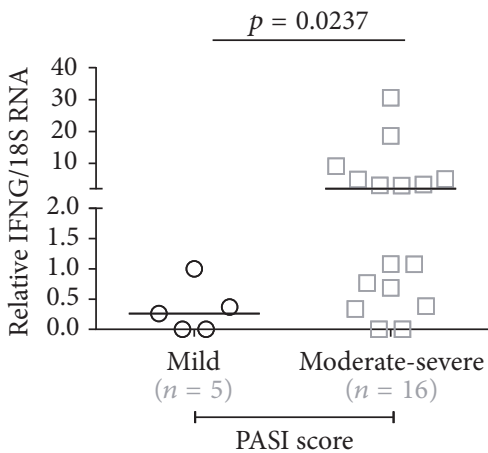

(b)

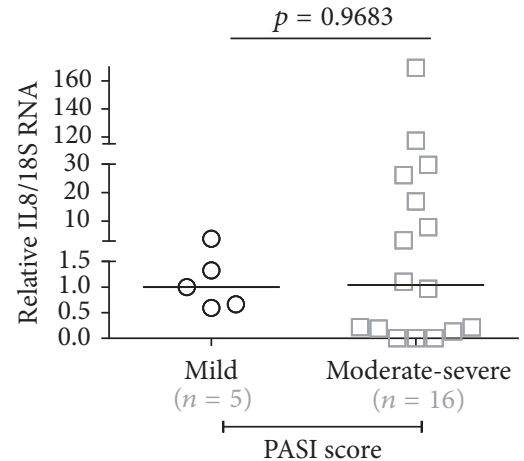

(e)

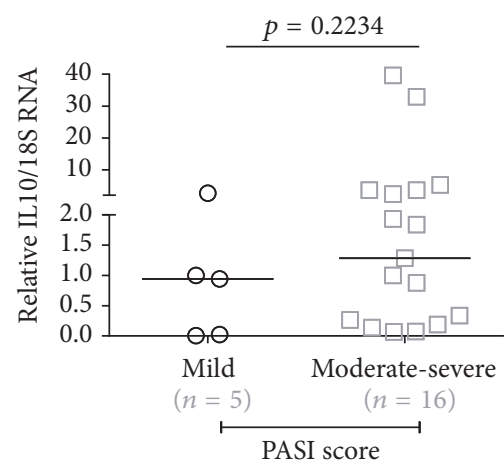

(h)

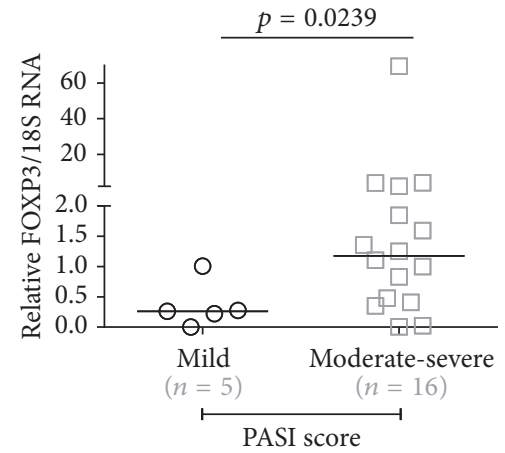

(c)

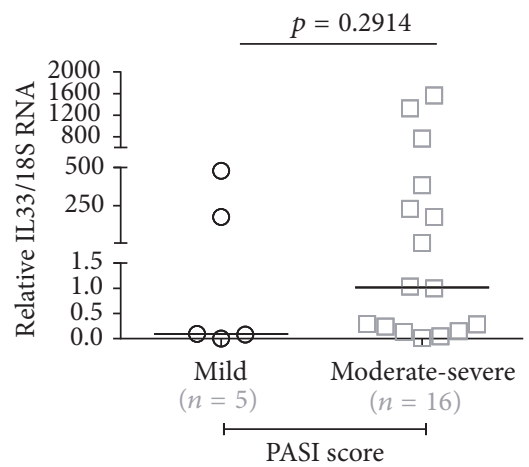

(f)

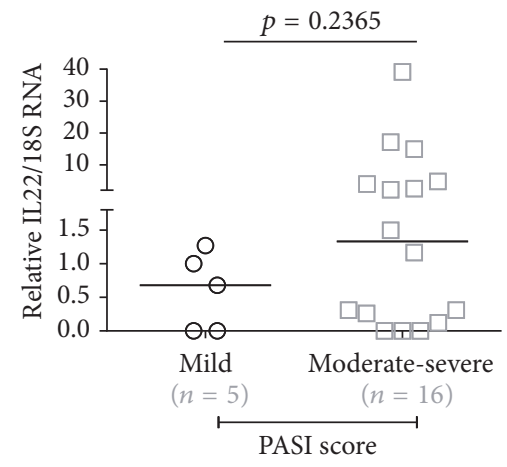

(i)

Figure 1: Transcripts levels of (a) IL17A, (b) IFNG, (c) FOXP3, (d) IL36A, (e) IL8, (f) IL33, (g) RORC, (h) IL10, and (i) IL22 according to PASI's severity disease.

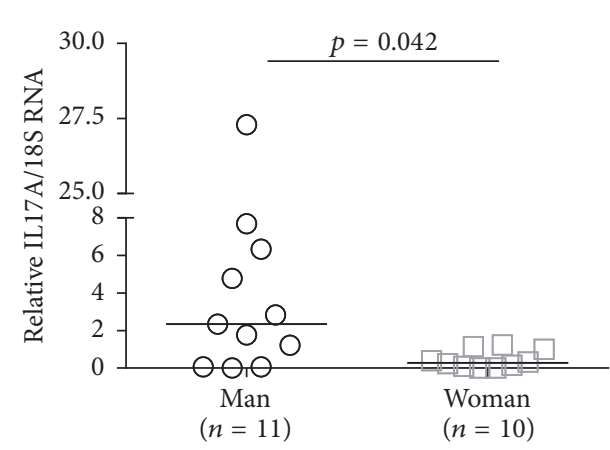

(a)

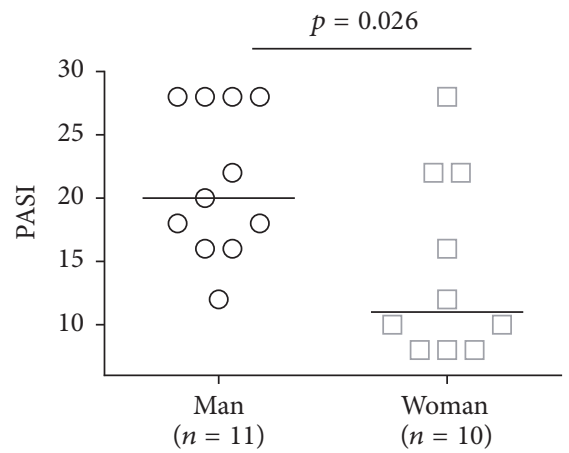

(b)

FIGURE 2: Different IL17A biopsy profile expression (a) and disease severity (b) between man and woman. 
TABLE 2: Interplay among cytokines in human psoriasis lesions ${ }^{\mathrm{a}}$.

\begin{tabular}{|c|c|c|c|c|c|c|c|c|c|}
\hline & IL8 & IL-33 & IL36A & IL17A & FOXP3 & RORC & IL22 & IFNG & IL10 \\
\hline \multirow{2}{*}{ IL8 } & & $p=0.443$ & $p=0.006$ & $\mathrm{p}=0.037^{*}$ & $p=0.064$ & $p=0.798$ & $\mathrm{p}=0.044^{*}$ & $p=0.087$ & $\mathrm{p}=0.039^{*}$ \\
\hline & & $r=-0.176$ & $r=0.574$ & $\mathbf{r}=0.457$ & $r=0.410$ & $r=0.059$ & $\mathrm{r}=0.442$ & $r=0.382$ & $\mathbf{r}=0.452$ \\
\hline \multirow{2}{*}{ IL33 } & $p=0.443$ & & $p=0.106$ & $p=0.126$ & $p=0.097$ & $p=0.278$ & $p=0.857$ & $p=0.279$ & $p=0.211$ \\
\hline & $r=-0.176$ & & $r=0.362$ & $r=0.344$ & $r=0.371$ & $r=0.248$ & $r=-0.041$ & $r=0.247$ & $r=0.284$ \\
\hline \multirow{2}{*}{ IL36A } & $\mathrm{p}=0.006^{* *}$ & $p=0.106$ & & $\mathrm{p}=0.001^{* *}$ & $\mathrm{p}<0.001^{* * *}$ & $p=0.096$ & $p=0.117$ & $\mathbf{p}=0.007^{* *}$ & $\mathbf{p}=0.002^{* *}$ \\
\hline & $r=0.574$ & $r=0.362$ & & $r=0.651$ & $\mathbf{r}=\mathbf{0 . 7 7 4}$ & $r=0.372$ & $r=0.351$ & $r=0.563$ & $\mathbf{r}=0.622$ \\
\hline \multirow{2}{*}{ IL17A } & $p=0.037$ & $p=0.126$ & $p=0.001$ & & $\mathrm{p}<0.001^{* * *}$ & $\mathrm{p}=0.017^{*}$ & $\mathrm{p}=0.049^{*}$ & $\mathrm{p}=0.004^{* *}$ & $\mathbf{p}<0.001^{* * *}$ \\
\hline & $r=0.457$ & $r=0.344$ & $r=0.651$ & & $\mathbf{r}=0.697$ & $\mathrm{r}=0.511$ & $\mathrm{r}=\mathbf{0 . 4 3 4}$ & $\mathbf{r}=0.598$ & $\mathbf{r}=\mathbf{0 . 7 9 5}$ \\
\hline \multirow{2}{*}{ FOXP3 } & $p=0.064$ & $p=0.097$ & $\mathrm{p}<0.001^{* * *}$ & $\mathbf{p}<0.001^{* * *}$ & & $\mathbf{p}<0.001^{* * *}$ & $p=0.109$ & $\mathrm{p}=0.020^{*}$ & $p=0.006^{* *}$ \\
\hline & $r=0.410$ & $r=0.371$ & $\mathbf{r}=\mathbf{0 . 7 7 4}$ & $\mathbf{r}=\mathbf{0 . 6 9 7}$ & & $r=0.686$ & $r=0.359$ & $\mathbf{r}=\mathbf{0 . 5 0 0}$ & $\mathbf{r}=0.574$ \\
\hline \multirow{2}{*}{ RORC } & $p=0.798$ & $p=0.278$ & $p=0.096$ & $\mathbf{p}=0.017^{*}$ & $\mathrm{p}<0.001^{* * *}$ & & $p=0.821$ & $p=0.415$ & $\mathrm{p}=0.049^{*}$ \\
\hline & $r=0.059$ & $r=0.248$ & $r=0.372$ & $\mathbf{r}=0.511$ & $r=0.686$ & & $r=0.052$ & $r=0.187$ & $\mathrm{r}=\mathbf{0 . 4 3 3}$ \\
\hline \multirow{2}{*}{ IL22 } & $\mathrm{p}=\mathbf{0 . 0 4 4}$ & $p=0.857$ & $p=0.117$ & $\mathrm{p}=0.049^{*}$ & $p=0.109$ & $p=0.821$ & & $p=0.179$ & $\mathrm{p}=0.024^{*}$ \\
\hline & $\mathrm{r}=0.442$ & $r=-0.041$ & $r=0.351$ & $\mathrm{r}=\mathbf{0 . 4 3 4}$ & $r=0.359$ & $r=0.052$ & & $r=0.304$ & $\mathbf{r}=0.487$ \\
\hline \multirow{2}{*}{ IFNG } & $p=0.087$ & $p=0.279$ & $\mathrm{p}=0.007^{* *}$ & $\mathrm{p}=0.004^{* *}$ & $\mathrm{p}=0.020^{*}$ & $p=0.415$ & $p=0.179$ & & $\mathrm{p}=0.028^{*}$ \\
\hline & $r=0.382$ & $r=0.247$ & $\mathrm{r}=0.563$ & $\mathbf{r}=\mathbf{0 . 5 9 8}$ & $\mathbf{r}=0.500$ & $r=0.187$ & $r=0.304$ & & $r=0.476$ \\
\hline \multirow{2}{*}{ IL10 } & $\mathrm{p}=0.039^{*}$ & $p=0.211$ & $\mathbf{p}=0.002^{* *}$ & $\mathrm{p}<0.001^{* * *}$ & $\mathrm{p}=0.006^{* *}$ & $\mathrm{p}=0.049^{*}$ & $\mathrm{p}=0.024^{*}$ & $\mathrm{p}=0.028^{*}$ & \\
\hline & $\mathrm{r}=0.452$ & $r=0.284$ & $r=0.622$ & $\mathbf{r}=0.795$ & $\mathbf{r}=0.574$ & $\mathrm{r}=0.433$ & $\mathbf{r}=0.487$ & $\mathrm{r}=0.476$ & \\
\hline
\end{tabular}

${ }^{a}$ Determination of statistical correlations was made according to Spearman's rank correlation test and represented by $p$ value. $*$ equals $p<0.05, * * p<0.01$, and $* * * p<0.001$. The correlation coefficients are represented by " $r$."

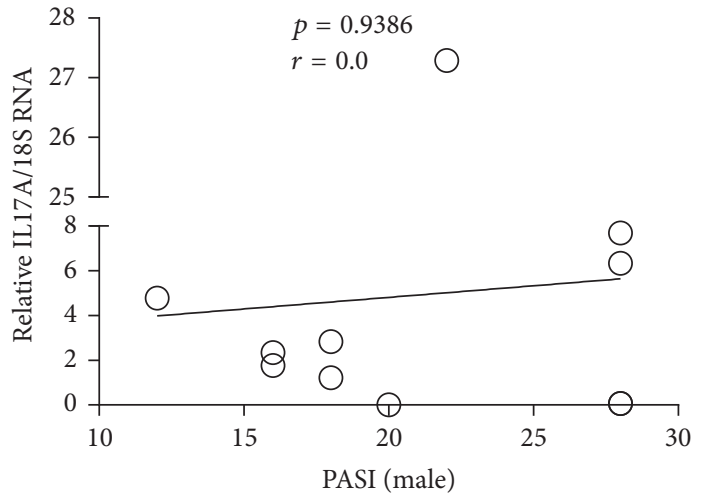

(a)

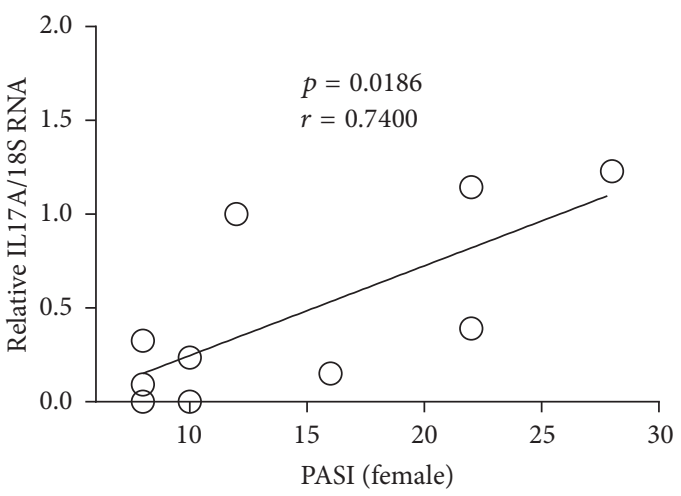

(b)

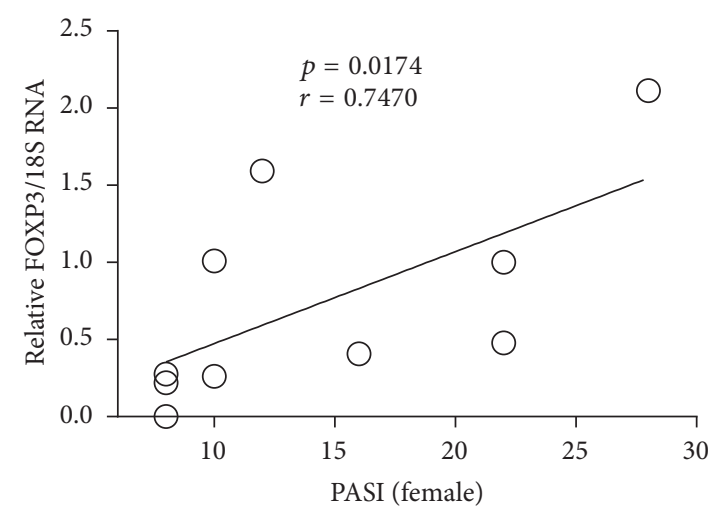

(c)

FIGURE 3: Correlation of IL17A (a, b) and FOXP3 (c) with disease severity between man and woman. 
TABLE 3: Correlation coefficients and $p$ values by gender ${ }^{\mathrm{a}}$.

\begin{tabular}{lccc}
\hline Gene & Man & Gene & Woman \\
\hline IL17A vs IL8 & $p=0.017$ & IL17A vs IFNG & $p<0.001$ \\
& $r=0.716$ & & $r=0.976$ \\
IL17A vs IL22 & $p=0.041$ & IL17A vs FOXP3 & $p=0.049$ \\
& $r=0.633$ & & $r=0.644$ \\
IL17A vs IL36A & $p=0.031$ & FOXP3 vs IL36A & $p<0.001$ \\
& $r=0.664$ & & $r=0.806$ \\
IL17A vs IL10 & $p=0.001$ & - & - \\
IL10 vs IL8 & $r=0.882$ & - & - \\
IL10 vs IL22 & $p=0.042$ & - & - \\
IL36A vs IL8 & $r=0.633$ & & - \\
& $p=0.034$ & & - \\
RORC vs FOXP3 & $p=0.651$ & - & - \\
& $r=0.024$ & & - \\
\hline
\end{tabular}

${ }^{a}$ Determination of statistical correlations was made according to Spearman's rank correlation test and represented by $p$ value. $p<0.05, p<0.01$, and $p<0.001$. The correlation coefficient is represented by " $r$." "vs" means versus.

TABLE 4: Association of transcripts expression and disease activity ${ }^{\mathrm{a}}$.

\begin{tabular}{lccc}
\hline PASI score & Odds ratio & $95 \% \mathrm{CI}^{+}$ & $p$ value \\
\hline IL22 & 0.189 & $0.005-6.247$ & 0.351 \\
RORC & 0.388 & $0.053-2.825$ & 0.350 \\
FOXP3 & 0.014 & $6.18 \mathrm{e}-07-344.728$ & 0.411 \\
IL17A & $8.16 \mathrm{e}-06$ & $5.49 \mathrm{e}-10-0.121$ & 0.017 \\
IL36A & 21.499 & $0.191-2414.25$ & 0.203 \\
IL33 & 1.000 & $0.989-1.012$ & 0.881 \\
IL8 & 1.686 & $0.984-2.888$ & 0.057 \\
\hline
\end{tabular}

${ }^{\mathrm{a}} \mathrm{CI}^{+}$: confidence interval.

Further, we verified significant levels of FOXP3 in psoriasis lesions from severe patients. Considering that it is reported as a $\mathrm{T}$ regulatory cell transcription factor, we did not expect this increase. Soler and McCormick (2011) demonstrated that psoriasis patients really had regulatory $\mathrm{T}$ cells presenting FOXP3, although most of them were nonfunctional [29].

We did not observe significant differences in expression of IL8, IL22, IL10, RORC, IL33, and IL36A. However, we detected their signature in psoriatic skin at a transcript level and this corroborated with previous studies[9, 30,31].

Continuing our analysis, we conducted all possible correlations between cytokines. Then, we found statistically significant correlation between cytokines from all three pathways with each other. It is important to notice that IL17A showed correlation with almost all analysed transcripts. Carrier and colleagues (2011) previously identified the interregulation among Th17 cytokines and IL-36 homologous forms. They also verified significance in correlation between IL36A versus IL17A and IFNG [32], as we did.

Curiously, we noted that IL17A had strong significant correlation with anti-inflammatory FOXP3 and IL10. We did not expect increased FOXP3 transcripts in moderate-severe patients, since this correlation seems antagonistic. Nevertheless, recent studies demonstrated another side of Treg cells. Bovenschen et al. (2011) found positive triple $\mathrm{CD} 4^{+}$ $\mathrm{IL}^{-17^{+}} \mathrm{FOXP}^{+}$in lesions from psoriasis patients indicating copathogenic profile. Soler and colleagues (2013) also pointed the connection between IL17 and FOXP3. They defended that Treg cells in psoriasis readily turn into IL-17-expressing cells $[33,34]$.

We also verified that when we considered gender, some correlations between cytokines only occurred in one of the groups. The male group preserved most of correlations especially those involving Th17 pathway. We could associate it to the fact that male group showed PASI mean $21.3 \pm 5.8$ and it is statistically higher than in women PASI $14.4 \pm 7.2$. It is worth pointing out that the severity of psoriasis in female patients may fluctuate with hormonal changes with worsening in puberty and peak at menopause [35]. Taking into consideration that population in this study consisted of eldered women $(57.7 \pm 9.8)$, they presented milder disease.

It is important to recognize our limitations in this study. We identified some failures such as small sample size, elevated cases of patients with moderate-severe disease, absence of healthy controls, and lack of proteomic analysis to confirm our findings. However, we know that, in psoriasis research, there are few studies with emphasis on experimental immunology in Brazil. Therefore, we hope that these data may open doors for future investigations.

\section{Conclusion}

Our study revealed increased transcripts levels of IL17A, IFNG, and FOXP3 in moderate-severe psoriasis patients. IL17A seems to play major influence in disease severity. In addition, we also showed complex network correlations between Th1, Th17, Treg, and IL-1 family related transcripts.

\section{Competing Interests}

All authors declare no conflict of interests.

\section{Acknowledgments}

The present study was supported by grants from Instituto Nacional de Ciência e Tecnologia para Inovação Farmacêutica (573663/2008-4), Coordenação de Aperfeiçoamento de Pessoal de Nível Superior (CAPES), Fundação de Amparo à Ciência e Tecnologia de Pernambuco (FACEPE), and Conselho Nacional de Desenvolvimento Científico e Tecnológico (CNPq).

\section{References}

[1] J. E. Gudjonsson and J. T. Elder, "Psoriasis: epidemiology," Clinics in Dermatology, vol. 25, no. 6, pp. 535-546, 2007.

[2] I. G. R. Baeta, F. V. Bittencourt, B. Gontijo, and E. M. A. Goulart, "Comorbidities and cardiovascular risk factors in patients with psoriasis," Anais Brasileiros de Dermatologia, vol. 89, no. 5, pp. 735-744, 2014. 
[3] H. S. Park, S. J. Koh, G. Y. Park et al., "Psoriasis concurrent with inflammatory bowel disease," Journal of the European Academy of Dermatology and Venereology, vol. 28, no. 11, pp. 1436-1441, 2014.

[4] W. Lew, A. M. Bowcock, and J. G. Krueger, "Psoriasis vulgaris: cutaneous lymphoid tissue supports T-cell activation and 'Type 1 ' inflammatory gene expression," Trends in Immunology, vol. 25, no. 6, pp. 295-305, 2004.

[5] V. Athie-Morales, H. H. Smits, D. A. Cantrell, and C. M. U. Hilkens, "Sustained IL-12 signaling is required for Th1 development," The Journal of Immunology, vol. 172, no. 1, pp. 6169, 2004.

[6] M. A. Lowes, C. B. Russell, D. A. Martin, J. E. Towne, and J. G. Krueger, "The IL-23/T17 pathogenic axis in psoriasis is amplified by keratinocyte responses," Trends in Immunology, vol. 34, no. 4, pp. 174-181, 2013.

[7] H. Duan, T. Koga, F. Kohda, H. Hara, K. Urabe, and M. Furue, "Interleukin-8-positive neutrophils in psoriasis," Journal of Dermatological Science, vol. 26, no. 2, pp. 119-124, 2001.

[8] J. Schmitz, A. Owyang, E. Oldham et al., "IL-33, an interleukin1-like cytokine that signals via the IL-1 receptor-related protein ST2 and induces T helper type 2-associated cytokines," Immunity, vol. 23, no. 5, pp. 479-490, 2005.

[9] A. Balato, S. Lembo, M. Mattii et al., "IL-33 is secreted by psoriatic keratinocytes and induces pro-inflammatory cytokines via keratinocyte and mast cell activation," Experimental Dermatology, vol. 21, no. 11, pp. 892-894, 2012.

[10] A. Balato, R. Di Caprio, L. Canta et al., "IL-33 is regulated by TNF- $\alpha$ in normal and psoriatic skin," Archives of Dermatological Research, vol. 306, no. 3, pp. 299-304, 2014.

[11] S. Vigne, G. Palmer, C. Lamacchia et al., "IL-36R ligands are potent regulators of dendritic and T cells," Blood, vol. 118, no. 22, pp. 5813-5823, 2011.

[12] S. Marrakchiet, P. Guigue, B. R. Renshaw et al., "Interleukin36-receptor antagonist deficiency and generalized pustular psoriasis," The New England Journal of Medicine, vol. 365, no. 7, pp. 620-628, 2011.

[13] L. Tortola, E. Rosenwald, B. Abel et al., "Psoriasiform dermatitis is driven by IL-36-mediated DC-keratinocyte crosstalk," The Journal of Clinical Investigation, vol. 122, no. 11, pp. 3965-3976, 2012.

[14] A. G. Richetta, C. Mattozzi, M. Salvi et al., "CD4+ CD25+ Tregulatory cells in psoriasis. Correlation between their numbers and biologics-induced clinical improvement," European Journal of Dermatology, vol. 21, no. 3, pp. 344-348, 2011.

[15] D. C. Soler, H. Sugiyama, A. B. Young, J. V. Massari, T. S. McCormick, and K. D. Cooper, "Psoriasis patients exhibit impairment of the high potency CCR $5^{+} \mathrm{T}$ regulatory cell subset," Clinical Immunology, vol. 149, no. 1, pp. 111-118, 2013.

[16] M. Friedrich, W.-D. Döcke, A. Klein et al., "Immunomodulation by interleukin-10 therapy decreases the incidence of relapse and prolongs the relapse-free interval in psoriasis," Journal of Investigative Dermatology, vol. 118, no. 4, pp. 672-677, 2002.

[17] F. O. Nestle, D. H. Kaplan, and J. Barker, "Mechanisms of disease: Psoriasis," The New England Journal of Medicine, vol. 361, no. 5, pp. 444-509, 2009.

[18] A. Menter, N. J. Korman, C. A. Elmets, S. R. Feldman, J. M. Gelfand, and K. B. Gordon, "Guidelines of care for the management of psoriasis and psoriatic arthritis," Journal of American Academy of Dermatology, vol. 58, no. 5, pp. 826-864, 2008.
[19] P. S. S. D. Oliveira, P. R. G. Cardoso, E. V. D. A. Lima et al., "IL-17A, IL-22, IL-6, and IL-21 serum levels in plaque-type psoriasis in Brazilian patients," Mediators of Inflammation, vol. 2015, Article ID 819149, 5 pages, 2015.

[20] M. B. M. Teunissen, C. W. Koomen, R. De Waal Malefyt, E. A. Wierenga, and J. D. Bos, "Interleukin-17 and interferon$\gamma$ synergize in the enhancement of proinflammatory cytokine production by human keratinocytes," Journal of Investigative Dermatology, vol. 111, no. 4, pp. 645-649, 1998.

[21] J. Li, D. Li, and Z. Tan, "The expression of interleukin17 , interferon $-\gamma$, and macrophage inflammatory protein-3 $\alpha$ mRNA in patients with psoriasis vulgaris," Journal of Huazhong University of Science and Technology, vol. 24, no. 3, pp. 294-296, 2004.

[22] C. Johansen, P. A. Usher, R. B. Kjellerup, D. Lundsgaard, L. Iversen, and K. Kragballe, "Characterization of the interleukin17 isoforms and receptors in lesional psoriatic skin," British Journal of Dermatology, vol. 160, no. 2, pp. 319-324, 2009.

[23] M. A. Lowes, T. Kikuchi, J. Fuentes-Duculan et al., "Psoriasis vulgaris lesions contain discrete populations of Thl and Th17 T cells," Journal of Investigative Dermatology, vol. 128, no. 5, pp. 1207-1211, 2008.

[24] A. Jabbari, M. Suárez-Farĩas, S. Dewell, and J. G. Krueger, "Transcriptional profiling of psoriasis using RNA-seq reveals previously unidentified differentially expressed genes," Journal of Investigative Dermatology, vol. 132, no. 1, pp. 246-249, 2012.

[25] C. E. Joyce, X. Zhou, J. Xia et al., "Deep sequencing of small RNAs from human skin reveals major alterations in the psoriasis miRNAome," Human Molecular Genetics, vol. 20, no. 20, pp. 4025-4040, 2011.

[26] J. E. Gudjonsson, J. Ding, A. Johnston et al., "Assessment of the psoriatic transcriptome in a large sample: additional regulated genes and comparisons with in vitro models," Journal of Investigative Dermatology, vol. 130, no. 7, pp. 1829-1840, 2010.

[27] J. Kim, C.-H. Oh, J. Jeon et al., "Molecular phenotyping small (Asian) versus large (Western) plaque psoriasis shows common activation of IL-17 pathway genes but different regulatory gene sets," Journal of Investigative Dermatology, vol. 136, pp. 161-172, 2016.

[28] M. Suárez-Fariñas, K. Li, J. Fuentes-Duculan, K. Hayden, C. Brodmerkel, and J. G. Krueger, "Expanding the psoriasis disease profile: interrogation of the skin and serum of patients with moderate-to-severe psoriasis," Journal of Investigative Dermatology, vol. 132, no. 11, pp. 2552-2564, 2012.

[29] D. C. Soler and T. S. McCormick, "The dark side of regulatory T cells in psoriasis," Journal of Investigative Dermatology, vol. 131, no. 9, pp. 1785-1786, 2011.

[30] K. Asadullah, W. Sterry, K. Stephanek et al., "IL-10 is a key cytokine in psoriasis," The Journal of Clinical Investigation, vol. 101, no. 4, pp. 783-794, 1998.

[31] K. Boniface, E. Guignouard, N. Pedretti et al., "A role for T cellderived interleukin 22 in psoriatic skin inflammation," Clinical and Experimental Immunology, vol. 150, no. 3, pp. 407-415, 2007.

[32] Y. Carrier, H.-L. Ma, H. E. Ramon et al., "Inter-regulation of Th17 cytokines and the IL-36 cytokines in vitro and in vivo: implications in psoriasis pathogenesis," Journal of Investigative Dermatology, vol. 131, no. 12, pp. 2428-2437, 2011.

[33] H. J. Bovenschen, P. C. Van De Kerkhof, P. E. Van Erp, R. Woestenenk, I. Joosten, and H. J. P. M. Koenen, "Foxp3+ regulatory $\mathrm{T}$ cells of psoriasis patients easily differentiate into IL-17A-producing cells and are found in lesional skin," Journal of Investigative Dermatology, vol. 131, no. 9, pp. 1853-1860, 2011. 
[34] D. C. Soler, H. Sugiyama, A. B. Young, J. V. Massari, T. S. McCormick, and K. D. Cooper, "Psoriasis patients exhibit impairment of the high potency CCR5+ T regulatory cell subset," Clinical Immunology, vol. 149, no. 1, pp. 111-118, 2013.

[35] R. Ceovic, M. Mance, Z. B. Mokos, M. Svetec, K. Kostovic, and D. S. Buzina, "Psoriasis: female skin changes in various hormonal stages throughout life-puberty, pregnancy, and menopause," BioMed Research International, vol. 2013, Article ID 571912, 6 pages, 2013. 


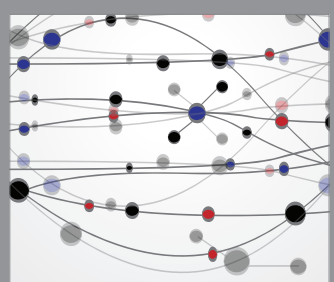

The Scientific World Journal
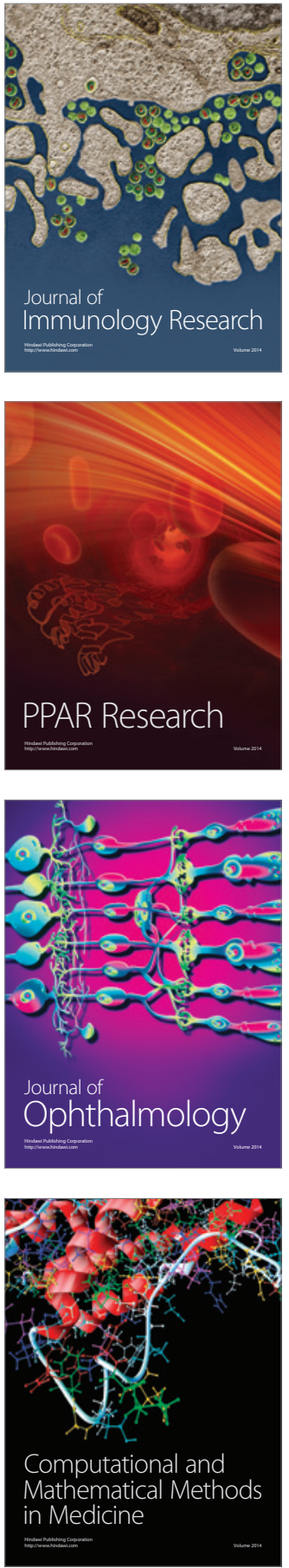

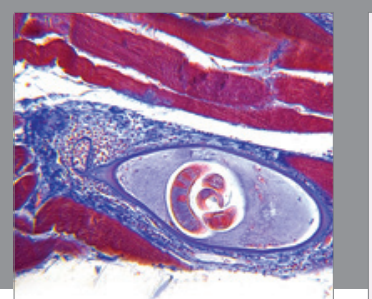

Gastroenterology Research and Practice

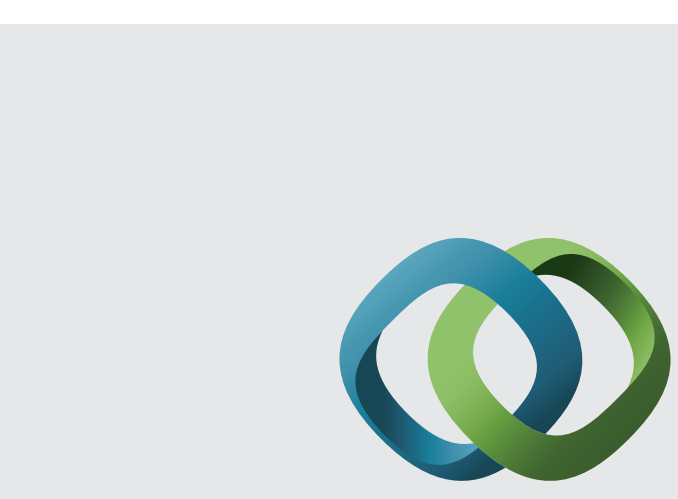

\section{Hindawi}

Submit your manuscripts at

http://www.hindawi.com
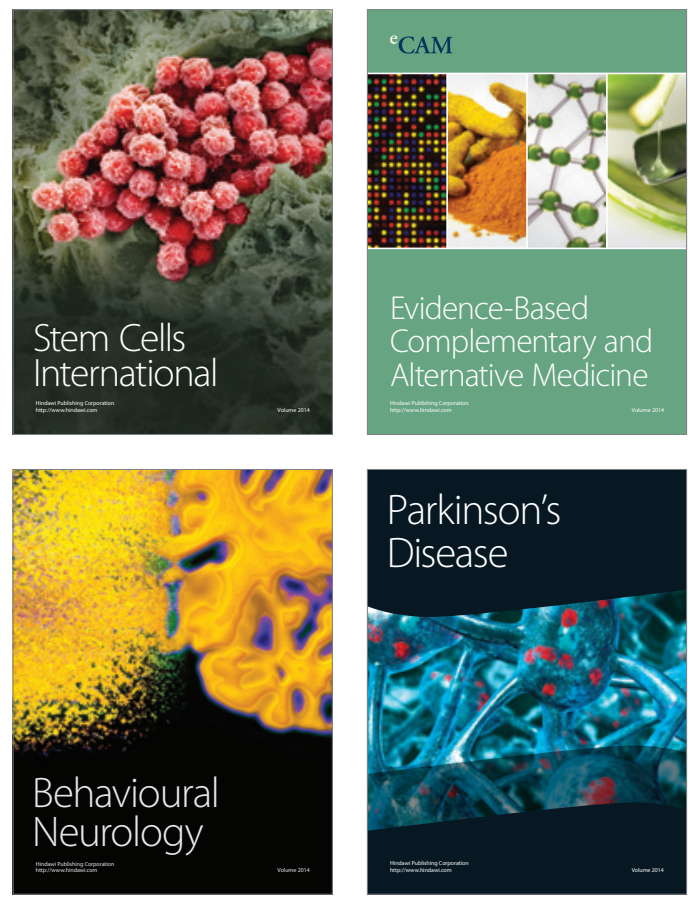
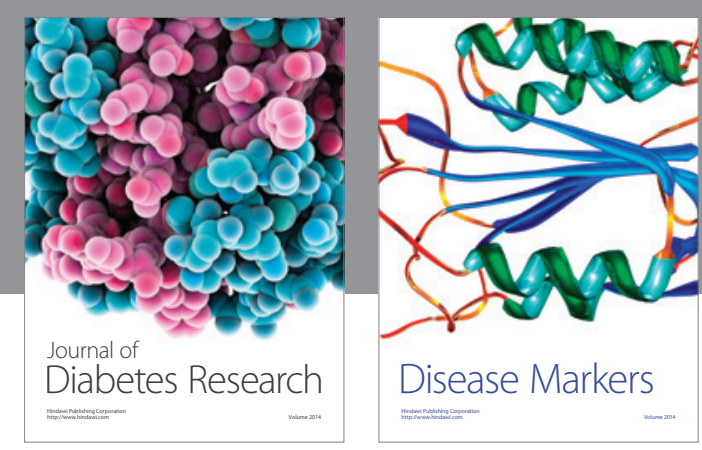

Disease Markers
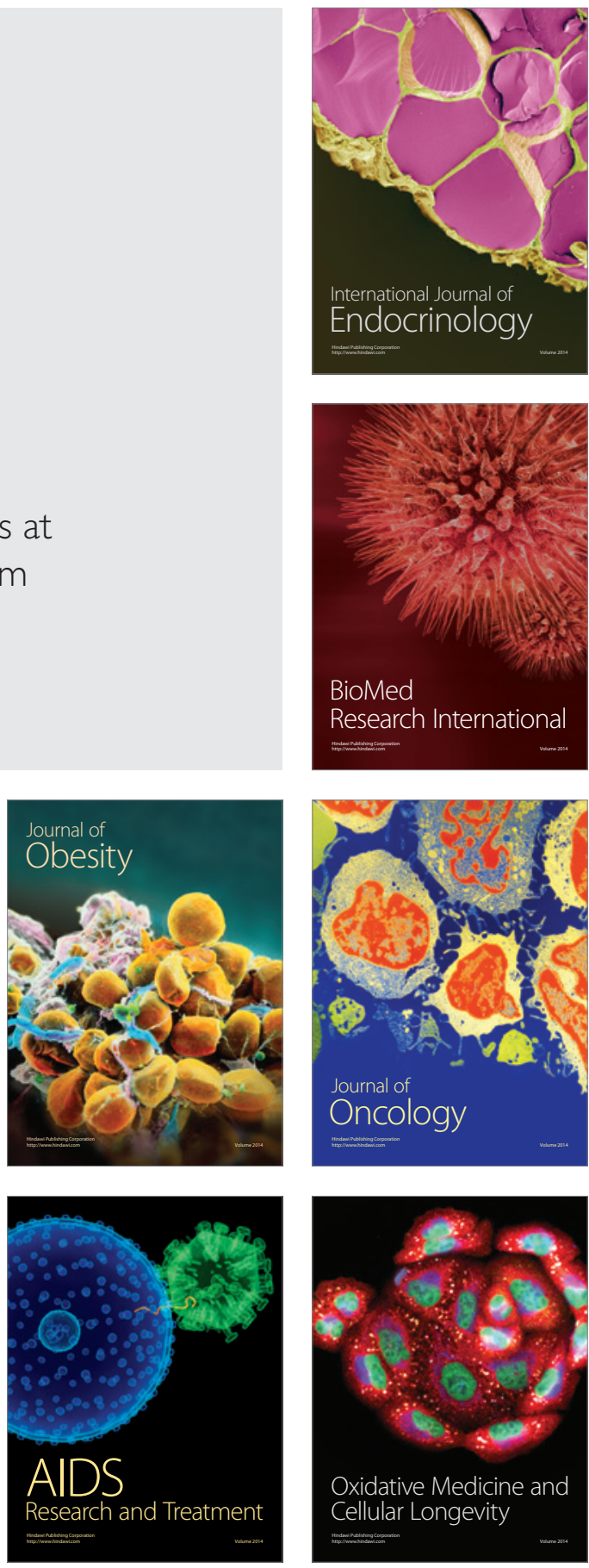